\title{
Headache Abstracts and Citations
}

\author{
Wade M. Cooper, DO \\ Robert G. Kaniecki, MD \\ Frederick R. Taylor, MD FAAN FAHS \\ Corresponding Author: \\ Wade Cooper \\ Email: wadecoop@med.umich.edu
}
Hitomi S, Kross K, Kurose M, Porreca F, Meng ID. Activation of dura-sensitive trigeminal neurons and increased c-Fos protein induced by morphine withdrawal in the rostral ventromedial medulla. Cephalalgia. 2017;37:407-417.

\begin{abstract}
Aims. Overuse of medications used to treat migraine headache can increase the frequency of headaches. Sudden abstinence from migraine medication can also lead to a period of withdrawal-induced headaches. The aim of this study was to examine the effect of morphine withdrawal localized to the rostral ventromedial medulla (RVM) on the activity of dura-sensitive spinal trigeminal nucleus caudalis (Vc) neurons. Methods. Rats were implanted with either morphine or placebo pellets for six to seven days before the microinjection of naloxone methiodide or phosphate-buffered saline into the RVM in urethane-anesthetized animals. Durasensitive neurons were recorded in the Vc and the production of c-Fos-like immunoreactivity was quantified. Results. In chronic morphine-treated animals, naloxone methiodide microinjections produced a significant increase both in ongoing and facial heat-evoked activity and an increase in Fos-positive neurons in the Vc and in the nucleus reticularis dorsalis, a brainstem region involved in diffuse noxious inhibitory controls. Conclusions. These results indicate that activation of pronociceptive neurons in the RVM under conditions of morphine withdrawal can increase the activity of neurons that transmit headache pain. Modulation of the subnucleus reticularis dorsalis by the RVM may explain the attenuation of conditioned pain modulation in patients with chronic headache.
\end{abstract}

Comments. Morphine has been used to model opioid induced hyperalgesia, in which neurons in the rostral ventromedial medulla mediate nociceptive neurons within the spinal trigeminal nucleus caudalis. ${ }^{l}$ This may result in hypersensitivity of the dura to both mechanical and temperature stimuli. Opioid exposure can increase pronociceptive neuron activity and lesioning of the RVM results in suppressing the opioid induced hyperalgesia. ${ }^{2}$ A relative imbalance between descending inhibition and facilitation has been implicated in opioid induced hyperalgesia and medication overuse headache. In this study, morphine was used to simulate opioid tolerance followed by a dose of mu opioid receptor antagonist directly into the rostral ventromedial medulla to represent cessation of opioid therapy. A series of electrophysiology studies were conducted to examine the effects of acute opioid withdrawal at the level of the RVM. The first finding in this study was an increase in both persistent and temperature evoked nociceptive signaling. Secondly, the presence of increased C-FOS positive neurons within the trigeminal nucleus caudalis indicates an increase in descending pain facilitation. These findings implicate the RVM as a possible generator for pain and headache. Additionally, the effects of naloxone injection to the RVM suggest a source for opioid withdrawal hyperalgesia.

Medication overuse headache via opioid medication can be quite challenging in a

This is the author manuscript accepted for publication and has undergone full peer review but has not been through the copyediting, typesetting, pagination and proofreading process, which may lead to differences between this version and the Version record. Please cite this article as doi:10.1111/ head.13152. 
clinical setting. Commonly prescribed short acting opioid agonists such as hydrocodone provide an opioid half-life of approximately 4 hours, meaning patients experience frequent peak and trough blood levels from this medication throughout the day. It is possible patients experience relative "withdrawals" several times per day based on these pharmacokinetic dynamics. This article suggests the chronic exposure of opioid medication may disrupt the balance of ascending and descending pain pathways at the level of the RVM as a mechanism of medication overuse headache.

1. Suzuki R, Porreca F, Dickenson AH. Evidence for spinal dorsal horn hyperexcitability in rats following sustained morphine exposure. Neurosci Lett 2006; 407:156-161.

2. Okada-Ogawa A, Porreca F, Meng ID. Sustained morphine-induced sensitization and

loss of diffuse noxious inhibitory controls in dura-sensitive medullary dorsal horn neurons. J Neurosci 2009; 29: 15828-15835.

-Wade M. Cooper, DO

FRT, are you still occasionally experiencing Mark Stillman flashbacks? WMC with his pain background is cut from the same cloth - starting us off with a first-pitch 100MPH basic science fastball just outside our clinical strike zones. Now with a baseball analogy I am having a Stew Tepper flashback. I will indeed take a swing at this abstract, and if I miss I suspect FRT will indicate the flaws in my technique. Modulation of brainstem antinociceptive circuitry is an interesting area for research into both opioid-induced hyperalgesia and medication overuse headache (MOH). ${ }^{1,2}$ From the rats of Hitomi et al I believe there is some clinical relevance to appreciate, but not as much as the authors or WMC would lead us to believe. I agree the RVM connections to the TNC and the changes associated with opioid exposure and withdrawal provide an appealing avenue illuminating opioid-induced hyperalgesia. In question is the expanding use of this data as an explanation for MOH with opioids, or more critically $M O H$ with non-opioid agents. Since opioids have clear receptor-related activity within the RVM, while triptans as an example likely do not, I am reluctant to ascribe all MOH physiology to the mechanisms outlined by these authors.

1. Vanderah TW, Suenaga NM, Ossipov MH. Tonic descending facilitation from the rostral ventromedial medulla mediates opioid-induced abnormal pain and antinociceptive tolerance. J Neurosci 2001; 21: 279-286.

2. Heinricher MM, Tavares I, Leith JL. Descending control of nociception: Specificity, recruitment and plasticity. Brain Res Rev 2009; 60: 214-225.

-Robert G. Kaniecki, MD

As Tiny Tim once said, "God bless Mark, God bless Stew, and Marcello, David and others, God bless all of the former editors of this abstract section." RGK you have me blessing and agreeing with you. While certainly the RVM plays the role stated here, opioid-induced hyperalgesia is a very distinct entity in my experience from that of medication overuse headache and while both opioids and serotonin pathways functionally facilitate pain through the RVM I am biased to think the mechanisms are distinct as well. ${ }^{1}$ 
1. Wei F, Dubner R, Zou S, et al. Molecular depletion of descending serotonin unmasks its novel facilitatory role in the development of persistent pain. J Neurosci. 2010;30:86248636. doi: 10.1523/JNEUROSCI.5389-09.2010.

-Frederick R. Taylor, MD

Akerman S, Simon B, Romero-Reyes M. Vagus nerve stimulation suppresses acute noxious activation of trigeminocervical neurons in animal models of primary headache. Neurobiol Dis. 2017;102:96-104.

Abstract Vagus nerve stimulation (VNS) has been reported to be effective in the abortive treatment of both migraine and cluster headache. Using validated animal models of acute duralintracranial (migraine-like) and trigeminal-autonomic (cluster-like) head pain we tested whether VNS suppresses ongoing and nociceptive-evoked firing of trigeminocervical neurons to explain its abortive effects in migraine and cluster headache. Unilateral VNS was applied invasively via hook electrodes placed on the vagus nerve. A single dose of ipsilateral or contralateral VNS, to trigeminal recording and dural-stimulating side, suppressed ongoing spontaneous and noxious dural-evoked trigeminocervical neuronal firing. This effect was dose-dependent, with two doses of ipsilateral VNS prolonging suppression of ongoing spontaneous firing (maximally by $\sim 60 \%$ ) for up to three hours, and dural-evoked (A $\delta$-fiber; by $\sim 22 \%$, C-fiber: by $\sim 55 \%$ ) responses for at least two hours. Statistically, there was no difference between ipsilateral and contralateral groups. Two doses of VNS also suppressed superior salivatory nucleus-evoked trigeminocervical neuronal responses (maximally by $\sim 22 \%$ ) for $2.5 \mathrm{~h}$, to model nociceptive activation of the trigeminal-autonomic pathway. VNS had no effect on normal somatosensory cutaneous facial responses throughout. These studies provide a mechanistic rationale for the observed benefits of VNS in the abortive treatment of migraine and cluster headache. In addition, they further validate these preclinical models as suitable approaches to optimize therapeutic efficacy, and provide an opportunity to hypothesize and dissect the neurobiological mechanisms of VNS in the treatment of primary headaches.

Comments. The vagus nerve includes sensory afferents that extend to headache related structures within the central nervous system including the nucleus solitarius, locus coeruleus, dorsal raphe nucleus and paraventricular hypothalamic nucleus. Additionally, the vagus nerve is also thought to have connections to the trigeminal nucleus caudalis and by extension the cervical spinal cord. The release of several neuropeptides have been linked to VNS including noradrenaline from the locus coeruleus, ${ }^{1}$ increase in of GABA in the CSF in epilepsy models. ${ }^{2,3}$ The inhibition of glutamate release from the trigeminal nucleus in a trigeminal pain model. ${ }^{4}$ In this model, hook electrodes were placed directly onto the vagus nerve and doses of electrical vagus nerve stimulation was able to suppress dural evoked nociceptive signaling in several key areas related to migraine. It seems the autonomic component of migraine and headache is 
receiving an increased amount of attention in the last several months. The arrival of peripheral nerve stimulators (not used in this research) targeting the vagus nerve sounds quite appealing. I am curious to hear my colleagues' opinion on this, as my very limited patient experience with peripheral vagus nerve stimulation for chronic headache has not been overwhelmingly positive.

1. Fornai F, Ruffoli R, Giorgi FS, Paparelli A. The role of locus coeruleus in the antiepileptic activity induced by vagus nerve stimulation. Eur J Neurosci. 2011;33:2169-2178.

2. Marrosu F, Serra A, Maleci A, Puligheddu M, Biggio G, Piga M. Correlation between $G A B A(A)$ receptor density and vagus nerve stimulation in individuals with drugresistant partial epilepsy. Epilepsy Res. 2003;55:59-70.

3. Ben-Menachem E, Hamberger A, Hedner T, et al. Effects of vagus nerve stimulation on amino acids and other metabolites in the CSF of patients with partial seizures. Epilepsy Res. 1995;20:221-227.

4. Oshinsky ML, Murphy AL, Hekierski H Jr, Cooper M, Simon BJ. Noninvasive vagus nerve stimulation as treatment for trigeminal allodynia. Pain. 2014;155:1037-1042.

-Wade M. Cooper, DO

Relief of acute migraine has been reported with vagal nerve stimulation (VNS) for approximately 15 years, with early reports arising from the primary use of this treatment in epilepsy. ${ }^{1}$ Recent clinical trials have established the use of VNS as effective in the treatment of acute migraine. ${ }^{2,3}$ Ackerman et al provide a neuroanatomical framework potentially responsible for the reported beneficial effects which, in animal models, suppressed ongoing spontaneous and noxious duralevoked trigeminocervical neuronal firing and superior salivatory nucleus-evoked trigeminocervical neuronal responses without impact on primary facial sensory neuronal function. Like WMC I have little clinical experience. It will be interesting to see how such a treatment behaves outside the artificial confines of a clinical trial.

1. Sadler RM, Purday RA, Rahey S. Vagal nerve stimulation aborts migraine in patient with intractable epilepsy. Cephalalgia 2002;22:482-484

2. Straube A, Ellrich J, Eren $O$, et al. Treatment of chronic migraine with transcutaneous stimulation of the auricular branch of the vagal nerve (auricular $t-V N S$ ): a randomized, monocentric clinical trial. J Headache Pain 2015;16:543. doi: 10.1186/s10194-0150543-3. Epub 2015 Jul 9

3. Puledda F, Goadsby P. An update on non-pharmacological neuromodulation for the acute and preventive treatment of migraine. Headache 2017;57:685-691.

-Robert G. Kaniecki, MD

It's always worthwhile to elucidate mechanisms. Outside a clinical trial indeed. VNS began as an invasive technique and has now become a percutaneous application. VNS would once upon a time have been prohibitively costly, will it become at least a cost-effective therapy if not at least commonly affordable?

-Frederick R. Taylor, MD 
Schulte LH, Allers A, May A. Hypothalamus as a mediator of chronic migraine: Evidence from high-resolution fMRI. Neurology. 2017;88:2011-2016.

Abstract OBJECTIVE: To identify pathophysiologic mechanisms of migraine chronification using a recently standardized protocol for high-resolution brainstem imaging of trigeminal nociceptive stimulation. METHODS: Eighteen episodic migraineurs (EMs), 17 chronic migraineurs (CMs), and 19 healthy controls (HCs) underwent painful ammonia stimulation of the left nostril in a 3T MRI scanner. Functional images were acquired with a brainstemoptimized protocol for high-resolution echo-planar imaging. RESULTS: We detected a significantly stronger activation of the anterior right hypothalamus in CMs compared to HCs. To exclude the headache as a prime mediator of the hypothalamic activations, we compared all migraineurs with headaches (EMs and CMs) with all migraineurs without headaches (EMs and $\mathrm{CMs}$ ) and HCs in a second analysis and found a more posterior region of the hypothalamus to be more activated bilaterally during headaches. CONCLUSIONS: Our data corroborate the fact that the hypothalamus plays a crucial role in the pathophysiology of migraine chronification and acute pain stage of migraineurs. While the more posterior part of the hypothalamus seems to be important for the acute pain stage, the more anterior part seems to play an important role in attack generation and migraine chronification.

Comments: The hypothalamus is a peanut sized structure that is famous for its role in maintaining homeostasis of multiple physiological systems within the body. It is the command center for several endocrine processes, and produces various hormones including vasopressin, corticotropin-releasing hormone, oxytocin, gonadotropin-releasing hormone, somatostatin, growth hormone-releasing hormone, and thyrotropin releasing hormone. The hypothalamus also influences emotional response through the limbic system, including the amygdala, thalamus, hippocampus, and olfactory cortex. Based on the likely origin of episodic migraine premonitory symptoms within the hypothalamus and previous evidence of hypothalamic activity in pain, these researchers sought to compare activity of the hypothalamus in those with episodic migraine, chronic migraine and healthy controls.

The findings from this study demonstrated hyperactivation of the posterior portion of the hypothalamus in acute pain, which is congruent with previous studies. ${ }^{1,2}$ Clinical symptoms of yawning or food craving prior to migraine seem to be hypothalamic in nature. The really interesting part of this study is the concept of chronification linked to the anterior aspect of the hypothalamus. The proposed mechanism is a disruption of the descending pain modulation system. The authors suggest repetitive stimulation from episodic migraine to result in a decreased threshold for anterior hypothalamic activation, resulting in a "generator" for increased migraine attacks and eventual progression to chronic migraine. The authors make a pitch for future studies in patients with medication overuse headache to explore the idea of medication overuse stimulating the same areas of the brain seen in migraine chronification without medication overuse or if there is a separate area of the brain with enhanced activity to imply an altogether different mechanism of increased headache frequency.

1. Denuelle M, Fabre N, Payoux P, Chollet F, Geraud G. Hypothalamic activation in spontaneous migraine attacks. Headache. 2007;47:1418-1426.

2. Maniyar FH, Sprenger T, Monteith T, Schankin C, Goadsby PJ. Brain activations in the 
premonitory phase of nitroglycerin-triggered migraine attacks. Brain. 2014;137:232241.

-Wade M. Cooper, DO

In these pages we have recently reviewed several articles implicating the hypothalamus as central to the physiology of acute migraine imply an altogether different mechanism of increased headache frequency. As WMC discusses above, the prodromal phase of migraine may directly result from activation of hypothalamic circuits. Photophobia during migraine may also be modulated, in part, by hypothalamic connections to the basal ganglia and thalamus. ${ }^{1}$ Another study recently investigated one migraine patient undergoing daily functional MRI brain scans for an entire month and found increased hypothalamic activation as a response to pain within 24 hours prior to headache onset as compared with the interictal state. Those authors showed increased pain-related hypothalamic functional connectivity to the spinal trigeminal nuclei during the preictal phase as compared with the interictal phase. ${ }^{2}$ These same authors recently published work that elegantly discusses the hypothesis that migraine does not arise from a single migraine generator, but rather from time-dependent changes in oscillating neurotransmitter networks that include hypothalamic and pontine structures. ${ }^{3}$ I highly recommend this article reviewed by WMC, as well as the third citation from Schulte.

1. Kagan R, Kainz V, Burstein R, Noseda R. Hypothalamic and basal ganglia projections to the posterior thalamus: possible role in modulation of migraine headache and photophobia. Neuroscience 2013; 248C:359-368.

2. Schulte LH, May A. The migraine generator revisited: continuous scanning of the migraine cycle over 30 days and three spontaneous attacks. Brain 2016;139:1987-1993.

3. Schulte L, May A. Of generators, networks, and migraine attacks. Curr Opin Neurol. 2017;30:241-245.

-Robert G. Kaniecki, MD

Agreed, another interesting read from this somewhat recent partnership of Schulte and May which may someday rival the productivity of Bigal and Lipton. One never knows. Migraine pathophysiologic complexity is beginning to better match the clinical complexity we all witness day to day.

-Frederick R. Taylor, MD

Askari G, Nasiri M, Mozaffari-Khosravi H, Rezaie M, Bagheri-Bidakhavidi M, Sadeghi O. The effects of folic acid and pyridoxine supplementation on characteristics of migraine attacks in migraine patients with aura: A double-blind, randomized placebo-controlled, clinical trial. Nutrition. 2017;38:74-79.

Abstract OBJECTIVE: The aim of this study was to assess the effects of folic acid alone and in combination with pyridoxine on characteristics of migraine attacks in adult migraine patients with aura. METHODS: This double-blind, randomized placebo-controlled, clinical trial was 
conducted on 95 migraine patients with aura (age range 18-65 y) in Isfahan, Islamic Republic of Iran, in 2014. Patients were randomly allocated to receive folic acid $(5 \mathrm{mg} / \mathrm{d})$ plus pyridoxine $(80 \mathrm{mg} / \mathrm{d})$ or folic acid alone $(5 \mathrm{mg} / \mathrm{d})$ or placebo (lactose) for $3 \mathrm{mo}$. Characteristics of migraine attacks including headache severity, attacks frequency, duration, and headache diary results (HDRs) were obtained for each patient at baseline and at the end of the study. RESULTS: Folic acid plus pyridoxine intake resulted in a significant decrease compared with placebo in headache severity $(-2.71 \pm 0.08$ versus $-2.19 \pm 0.05 ; \mathrm{P}<0.001)$, attack frequency $(-3.35 \pm 0.09$ versus $2.73 \pm 0.05 ; \mathrm{P}<0.001)$, duration $(-7.25 \pm 0.17$ versus $-6.5 \pm 0.07 ; \mathrm{P}<0.001)$, and HDR $(-$ $74.15 \pm 0.2$ versus $-72.73 \pm 0.1 ; \mathrm{P}<0.001)$. Additionally, the reduction in these characteristics of migraine attacks in the folic acid plus pyridoxine group was significant compared with the group given folic acid alone $(\mathrm{P}<0.001)$. However, these beneficial effects of the combined supplement became nonsignificant for attack duration compared with the folic acid-only and placebo groups after controlling for confounders. Folic acid intake without pyridoxine did not lead to a significant decrease in characteristics of migraine attacks compared with placebo group. CONCLUSIONS: Supplementation of folic acid with pyridoxine could decrease the characteristics of migraine attacks including headache severity, attack frequency, and HDR; however, further studies are needed to shed light on the findings of the present study.

Comments: Folate (vitamin B9) is an essential vitamin that is present in many foods including vegetables (especially green leafy types), fruit, dairy products, eggs, nuts and sometimes beer. Foods known for their especially high levels of folate are avocado, spinach, asparagus, and Brussels sprouts. Similarly, pyridoxine (vitamin B6) is also found in vegetables, dairy products, and grains with notable high counts found in spinach, carrots, and peas. Pyridoxine is thought to contribute to myelin formation as well as the production of serotonin, norepinephrine and tryptophan. ${ }^{1}$ Deficiencies of both vitamins may be associated with fatigue, depression, anxiety, and pain. Although both vitamins are added into various foods to reduce chances of dietary deficiency, malabsorption of both vitamins may be seen in those with inflammatory bowel disease or possibly disrupted gut microbiota.

This abstract was a randomized, double blinded, placebo controlled trial with three arms including a group of folic acid supplementation alone and a group of combined folic acid and pyridoxine. The study was done in Iran with 95 subjects that had a clinical diagnosis of migraine with aura. The authors found improvements in migraine attack frequency and severity, which were significantly greater in the combined folic acid / pyridoxine group compared to both folic acid alone and placebo. The study could have been better powered, and 3 months seems a bit too short for meaningful outcomes, but overall implies vitamin B6 should receive more consideration. Migraine has well known comorbidities with inflammatory bowel disease and gastric motility dysfunction. It is possible this mechanism is related to the preventive effects of folic acid and B6 supplementation. Eat your spinach!

1. Calderón-Guzmán D, Hernández-Islas JL, Espitia-Vázquez I,et al. Pyridoxine, regardless of serotonin levels, increases production of 5-hydroxytryptophan in rat brain. Arch Med Res. 2004;35:271-274.

-Wade M. Cooper, DO

The use of nutraceuticals in the prevention of migraine headaches is appealing at several levels. Patients are more comfortable with natural measures, and therefore may be more compliant. 
Many migraine preventive drugs have both short-term and potential long-term side effects. Finally, the presently available means of migraine prevention are not particularly effective in a significant percentage of patients. So why pyridoxine and folate? Prior work reported that folic acid and pyridoxine supplementation can reduce migraine symptoms by affecting homocysteine levels. That work involved lower doses of pyridoxine (25mg) and folic acid. The present study compared 3 groups: patients received daily capsules containing $5 \mathrm{mg}$ folic acid and $80 \mathrm{mg}$ pyridoxine, capsules containing $5 \mathrm{mg}$ folic acid, or capsules containing lactose for 3 months. As outlined above modest improvements in migraine frequency and severity were identified in the pyridoxine plus folic acid group. The endpoints did not necessarily parallel most migraine preventive studies, and the study population was small. Tolerability was excellent. Interesting work that may be a consideration in the clinic.

1. Menon S, Lea RA, Roy B, Hanna M, Wee S, Haupt L. Genotypes of the MTHFR C677 T and MTRR A66 $G$ genes act independently to reduce migraine disability in response to vitamin supplementation. Pharmacogenet Genomics 2012;22:741-749

-Robert G. Kaniecki, MD

I am not an opponent of nutraceuticals for our patients and have written on both the evidence and the potential biological mechanisms in the past. ${ }^{1,2,3}$ So let me play some devil's advocate here. Eat your spinach from WMC warrants caution for oxalate renal stone formers; nothing like renal colic superseding colicky migraine. $R G K$ was, may I opine, uncharacteristically positive about this work. Indeed, for a three armed study the population was small and underpowered and lacked IHS prevention endpoint standards. And were the statistically significant differences likely clinically meaningful to the patients? I would have loved to see a responsiveness psychometric; i.e., the ability of a measure to detect clinically meaningful changes in headache symptoms that occur as a result of treatment. ${ }^{4} R G K$ is most definitely correct about currently available migraine prevention compounded by unacceptable costs at least in North America. Yet, while pyridoxine 100mg is available over the counter for under \$1 per month folate at $5000 \mathrm{mcg}$ (5mg) is not inexpensive at retail at between \$18-25 per month with relatively limited choices (based on my searches current and past). The cost of folate at this dosage is simply prohibitive for most patients, especially given lack of coverage by any insurance, whether Medicaid, Commercial (employer), Medicare, TriCare, etc. WMC is thus sage at writing that B6 should receive more consideration.

1. Evans RW, Taylor FR. Expert Opinion: "Natural" or alternative medications for migraine prevention. Headache. 2006;46:1012-1018.

2. Taylor FR. Headache prevention with complementary and alternative medicine. Headache. 2009;49:966-968.

3. Taylor FR. Nutraceuticals and headache: the biological basis. Headache. 2011;51:484501 .

4. Loder EW, Rizzoli P. Detecting meaningful changes in trials of headache treatments: which outcome measure is best? Cephalalgia. 2012;32:947-949

-Frederick R. Taylor, MD 


\begin{abstract}
Abdolahi M, Tafakhori A, Togha M, Okhovat AA, Siassi F, Eshraghian MR, Sedighiyan M, Djalali M, Mohammadzadeh Honarvar N, Djalali M. The synergistic effects of $\omega-3$ fatty acids and nano-curcumin supplementation on tumor necrosis factor (TNF)- $\alpha$ gene expression and serum level in migraine patients. Immunogenetics. 2017;69:371-378.
\end{abstract}

Abstract. Migraine is a destabilizing neuroinflammatory disorder characterized by recurrent headache attacks. Evidences show tumor necrosis factor (TNF)- $\alpha$ play a role in neuroimmunity pathogenesis of migraine. TNF- $\alpha$ increase prostanoid production, hyperexcitability of neurons, and nociceptor activation resulted in neuroinflammation and neurogenic pain. $\omega-3$ fatty acids and curcumin exert neuroprotective and anti-inflammatory effects via several mechanisms including suppression of TNF- $\alpha$ gene expression and its serum levels. The aim of this study is an evaluation of synergistic effects of $\omega-3$ fatty acids and nano-curcumin on TNF- $\alpha$ gene expression and serum levels in migraine patients. The present study performed as a clinical trial over a 2month period included 74 episodic migraine patients in 4 groups and received $\omega-3$ fatty acids, nano-curcumin, and combination of them or placebo. At the start and the end of the study, the gene expression of TNF- $\alpha$ and TNF- $\alpha$ serum levels was measured by real-time PCR and ELISA method, respectively. Our results showed that the combination of $\omega-3$ fatty acids and nanocurcumin downregulated TNF- $\alpha$ messenger RNA (mRNA) significantly in a synergistic manner $(\mathrm{P}<0.05)$. As relative to gene expression, a significant greater reduction in serum levels of TNF$\alpha$ were observed in the combination group, but no significant differences in other groups.

Supplementation with $\omega-3$ fatty acids or nano-curcumin alone did not show significant reduction either in mRNA or serum levels of TNF- $\alpha$. In addition, a much greater reduction in attack frequency was found in the combination group $(\mathrm{P}<0.001)$. These findings indicated that $\omega-3$ fatty acids and curcumin supplementation can be considered as a new promising approach in migraine management.

Comments. Curcumin has become a popular vitamin supplement for any symptom with a whiff of inflammation in the body. Most supplemental curcumin is derived from turmeric, which is part of the ginger family. This supplement hails from ayurvedic medicine roots (pun intended), a healing approach from ancient India. Curcumin is thought to have clinical effects within the body by reducing systemic inflammation. A recent meta-analysis has demonstrated turmeric supplementation to be related to reductions in serum $C$-reactive protein levels. ${ }^{1} T N F-\alpha$ is a pro inflammatory cytokine that is thought to be related to the activation of soon to be famous calcitonin gene related peptide (CGRP) and subsequent stimulation of the prostaglandin pathway. Migraine has been linked to the presence of TNF gene polymorphisms. ${ }^{2,3}$ The presence of TNF- $\alpha$ elevation within the cerebrospinal fluid has been linked to new daily persistent headache (NDPH) in a small patient series. ${ }^{4}$ Omega 3 fatty acids have also been linked to systemic inflammation. A recent publication has reported Omega 3 fatty acid supplementation to be effective in migraine prophylaxis as adjunctive therapy in patients using amitriptyline. ${ }^{5}$ However, my anecdotal observations in patients using omega 3 fatty acid supplementation for migraine has been disappointing. Admittedly I have not considered combination of curcumin and omega 3 for synergy in headache patients as reported in the selected abstract. This abstract had a population size of 74 patients, which were divided into 4 groups. This complexity substantially reduces the power of the study. I am not sure what to make of the results and am eager to hear the thoughts of my co-editors. 
1. Sahebkar A. Are curcuminoids effective C-reactive protein-lowering agents in clinical practice? Evidence from a meta-analysis. Phytother Res. 2014;28:633-642.

2. Chen M, Tang W, Hou L, et al. Tumor Necrosis Factor (TNF) -308G>A, Nitric Oxide Synthase 3 (NOS3) +894G>T Polymorphisms and Migraine Risk: A Meta-Analysis. PLoS One. 2015;10:e0129372.

3. Liu R, Ma M, Cui M,et al. Effects of tumor necrosis factor- $\beta$ (TNF- $\beta$ ) $252 A>G$ polymorphism on the development of migraine: a meta-analysis. PLoS One. 2014;9:e100189.

4. Rozen T, Swidan SZ. Elevation of CSF tumor necrosis factor alpha levels in new daily persistent headache and treatment refractory chronic migraine. Headache. 2007;47:1050-1055.

5. Soares AA, Louçana PM, Nasi EP, Sousa KM, Sá OM, Silva-Néto RP. A doubleblind, randomized, and placebo-controlled clinical trial with omega-3 polyunsaturated fatty acids (OPFA $\varpi-3)$ for the prevention of migraine in chronic migraine patients using amitriptyline. Nutr Neurosci. 2017;5:1-5.

-Wade M. Cooper, DO

Evidence for beneficial effects of omega-3 polyunsaturated fish oils (OPFAs) in migraine prevention has been lacking. A single class I randomized controlled trial examined the role of OPFAs in 196 patients with migraine. When compared to those taking placebo, those treated with 6 grams of OPFAs daily showed no significant reduction in headache attacks following 16 weeks of treatment. ${ }^{1}$ Per protocol analysis did display some significance, and the AHS and AAN guidelines assigned OPFAs a level $U$ designation (inadequate evidence to support or refute benefit). ${ }^{2}$ As with many nutraceutical therapies the harms appear limited, but even after the article provided by WMC benefits remain to be fully substantiated. Since FRT is clearly the expert among us in this arena, I now defer to him. ${ }^{3}$

1. Pradalier A, Bakouche P, Baudesson G. Failure of omega-3 polyunsaturated fatty acids in prevention of migraine: A double-blind study versus placebo. Cephalalgia. 2001;21:818-822.

2. Rajapakse T, Pringsheim T. Nutraceuticals in Migraine: A Summary of Existing Guidelines for Use. Headache. 2016;56:808-816

3. Taylor FR. Nutraceuticals and headache: The biological basis. Headache 2011;51:484501.

-Robert G. Kaniecki, MD

I may just be on the wrong side of good natured here, but haven't we got the cart before the horse. If I understand this study at all it wasn't a primary outcome study of efficacy, it was rather a proof of concept study regarding synergy for gene expression and serum inflammatory marker levels. Therefore the statement regarding attack frequency reduction is interesting at best. Regarding the primary outcomes only the combination produced desirable reductions in gene expression and serum inflammatory levels. It is therefore reasonable to suggest this neither supports nor negates the current research conclusion regarding failure of monotherapy with 
omega-3 polyunsaturated fatty acids (OPFAs) in migraine prevention. ${ }^{1,2}$ I have added additional research papers, which $R G K$ may have chosen not to reference for good reasons. Before comments of these add-ons let me note that the RGK referenced study, as he mentioned, used an adequate dose of OPFAs at 6 grams per day; at least based on my knowledge of any known research on OPFAs. The first add-on adolescent study fails to show statistical superiority of fish oil versus presumed placebo olive oil, but both with non-standard dramatic improvements in headache measures. ${ }^{1}$ In that study the supplementation dose was 2 grams per day which is definitely safe but only possibly adequate even considering age and on average lower body mass index than adults. However, without dose-response curves for OPFAs we really don't know whether this could have been too low a dose. The total EPA+DHA totaled $1254 \mathrm{mg}$ per day; again possibly subtherapeutic given the unknowns. Yet, I must add that for its time this was a definitely well-intended dose and even high by some study standards of today. The second was a fish oil add-on to sodium valproate with benefit over valproate alone in the first 4 weeks with benefit lost thereafter in a 12 week trial. ${ }^{2}$ This trial however only used 180mg OPFAs, most certainly a meaningless dose by today's standards and any legit research I am aware of in any field involving inflammatory diseases.

1. Harel Z, Gascon G, Riggs S, et al. Supplementation with omega-3 polyunsaturated fatty acids in the management of recurrent migraines in adolescents. J Adolesc Health. 2002;31:154-161.

2. Tajmirriahi M, Sohelipour M, Basiri K, et al. The effects of sodium valproate with fish oil supplementation or alone in migraine prevention: A randomized single-blind clinical trial. Iran J Neurol. 2012;11:21-24.

-Frederick R. Taylor, MD

\section{Müller KI, Alstadhaug KB, Bekkelund SI. Headache patients' satisfaction with telemedicine: a 12-month follow-up randomized non-inferiority trial. Eur J Neurol. 2017;24:807-815.}

Abstract BACKGROUND AND PURPOSE: We investigated non-acute headache patients' long-term satisfaction with a telemedicine consultation and consultation preferences in northern Norway. We hypothesized that patients were not less satisfied with telemedicine than traditional consultations. We also examined the influence of gender, age and education on satisfaction. METHODS: For 2.5 years, patients were consecutively screened, recruited and randomly assigned to telemedicine or traditional visits with a consultation at a neurological outpatient department. The primary endpoint was frequency of satisfied patients at 3 and 12 months. Secondary endpoints were satisfaction with consultation, communication, information, diagnosis, advice and prescriptions, and preferred visit form at 12 months. RESULTS: Of 402 participants, $279(69.4 \%)$ answered questionnaires at both 3 and 12 month, and 291 (72.4\%) responded at 12 months. The long-term satisfaction of telemedicine patients was 124/145 (85.5\%) compared with $118 / 134(88.1 \%)$ in the traditional group $(\mathrm{P}=0.653)$. The groups did not differ with respect to secondary endpoints, but females were more satisfied with telemedicine communication $(\mathrm{P}=$ 
0.027). In the telemedicine group, 99/147 (67.3\%) were indifferent to the type of consultation. Age and education did not alter the primary results. CONCLUSIONS: At 1 year after a specialist evaluation for headache, telemedicine patients did not express less satisfaction than those with traditional consultation. Telemedicine specialist consultations may be a good alternative for headache patients in secondary care.

Comments. Patient accessibility to headache specialists remains woefully poor in the United States, and likely worldwide. Many motivated patients may find themselves traveling far distances to be treated by providers who have expertise in the field. As headache medicine has the combination of high patient demand and limited physical exam input in many cases, telemedicine seems to make sense. This abstract evaluated patient satisfaction with telemedicine visits in place of traditional in office consultation. All subjects were referred by primary care physicians in Norway. Subjects with an abnormal neurologic examination noted by the referring physician were excluded from the study. The intervention was a one-time consultation without follow-up care or longitudinal management (Sounds appealing). The telemedicine evaluation included two-way audio and video communication. The outcomes speak for themselves. Those receiving telemedicine in place of traditional in office consultation had similar satisfaction at 12 months. Telemedicine is already commonly applied for other neurological disorders, most notably acute stroke management. Telemedicine for headache practices just makes logical sense. Unfortunately, logic and common sense is not applicable to $3^{\text {rd }}$ party payers, making reimbursement for telemedicine services hard to achieve. My business focused colleagues assure me this will change soon, and telemedicine will become a covered benefit for patients with traditional insurance. I guess we will soon find out.

-Wade M. Cooper, DO

In my opinion the initial patient visit - if geographically feasible - belongs in the office. Screening for secondary headaches requires a thorough neurological examination, and a great leap of faith is taken when we assume a busy primary care practice is effective in screening for subtle nuances of history or exam in all headache cases. Although the impact of telemedicine on isolated new patient consultations were specifically assessed by the authors and reviewed by $W M C$, I believe the optimal benefits for cyber visits will be maximized in return patient visits. The majority of those patients have primary headaches and relatively normal physical exams, and aside for pulse and blood pressure measurements (easily provided by the patient) I find that most of my management decisions involve conversations across my office desk. Why could they not then occur on my office desk? Numerous potential efficiencies are possible. Less time from home or work for the patient, who also saves travel and parking expenses. Lower overhead expense for the clinician (waiting room and exam room space) and likely a schedule which is less likely to involve late or no-show patients. Aside from the occasional system downtime or power outage, there may be few logistical problems with cyber visits. FRT?

-Robert G. Kaniecki, MD

On initial read of WMC and RGK comments I became aware I was nodding my head yes to both and wondering how could that be. I am a hands on (with permission) type of clinician who likes to feel neck and shoulder musculature, and see and "smell-so to speak" the patient. And I have 
done it my way for decades. I am naturally inclined then to negate the benefits of telemedicine so well outlined by my colleagues and extol the negatives, including RGK's concern for history nuances and exam subtleties. So I find myself agreeing with RGK that geographical feasibility makes sense warranting in-office appointment. Yet, I find the logical sense for telemedicine, for the reasons already put forth and more, gaining strength every time I return to think about it. For the patient even if nothing but presumably fewer hassles than an in-office appointment is gained their satisfaction is likely to soar. Additionally, the risks we providers all accept for negative patient evaluations handed or mailed to patient representatives because of "front-desk" or "patient-staging" encounters at least changes or morphed? In the end the reasoned RGK argument against a first time telemedicine headache encounter is a "... doth protest too much, methinks. "After all, stroke telemedicine encounters routinely occur with manifest and nuanced exam findings present with successful conclusions to a satisfactory degree based on the best the data has to offer. ${ }^{1}$ Maybe most importantly telestroke is cost-effective from both a societal and a hospital perspective. ${ }^{2}$ One should bet that teleheadache would be cost-effective from a societal perspective as well. RGK's nuanced headache questions can be asked via teleheadache and if a red flag encountered the appropriate steps readily engineered. As for the exam we may wish to suggest that exam subtleties require our eyes, mind and hands on the patient, but in reality the nuances of the neurologic exam rarely are the key to headache diagnosis. Telemedicine provides us with eyes and mind, and rather than hands (to temporal arteries for example) most often we need: 1) a good eye exam which increasingly can be assisted via smart phone technologies (albeit the biggest arguable exam component), 2) observation of the cognitive state of the patient (not at all likely superior in person than via telemedicine), 3) a walking-gait exam for integrated visual, sensory, motor and coordination health (again easily done by telemedicine). These three components are the key exam variables, not Babinski's, vibration or two point asymmetries, etc. I believe the key neurologic exam can be completed with tweaking via telemedicine. So while, in the room multi-sensory contact is lost (and I would proffer a huge loss for me) many patients will benefit in yet unaccounted for ways making telemedicine of potential enormous benefit for them. Yet telestroke evaluations tell us that the telemedicine intervention adds complexity for the patient which include aspects of staff interactions, cognitive participation, emotional reactions, and coherence (understanding) of care. ${ }^{l}$ So does this study under discussion really give us a sufficient evaluation or is it just the tip of an iceberg approach. I propose we will need considerably more information; to be sure. What will also be needed should RGK be correct in his concerns is a good patient outcomes study and an objective risk/benefit assessment for patient and practitioner alike. I hope benefit will microsize risk.

1. Wechsler LR, Demaerschalk BM, Schwamm LH, et al; American Heart Association Stroke Council; Council on Epidemiology and Prevention; Council on Quality of Care and Outcomes Research. Telemedicine Quality and Outcomes in Stroke: A Scientific Statement for Healthcare Professionals From the American Heart Association/American Stroke Association. Stroke. 2017;48:e3-e25. doi: 10.1161/STR.0000000000000114.

2. Nelson RE, Saltzman GM, Skalabrin EJ, Demaerschalk BM, MajersikJJ. The costeffectiveness of telestroke in the treatment of acute ischemic stroke. Neurology. 2011;77:1590-1598. doi: 10.1212/WNL.0b013e318234332d 
-Frederick R. Taylor, MD

\section{Eckner JT, Seifert T, Pescovitz A, Zeiger M, Kutcher JS. Is Migraine Headache Associated With Concussion in Athletes? A Case-Control Study. Clin J Sport Med. 2017;27:266-270.}

Abstract OBJECTIVE: The purpose of this study was to investigate the association between migraine headache and concussion in athletes. DESIGN: Case-control observational study. SETTING: A university-associated combined sports neurology and orthopedic sports medicine clinic. PARTICIPANTS: A total of 221 male $(n=140)$ and female $(n=81)$ athletes aged 12 to 24 years, including 115 concussion cases (52\%) and 106 orthopedic controls (48\%), were included in this study. INTERVENTIONS: Participants completed a one-page questionnaire that recorded their age, sex, reason for visit (concussion vs any other injury), concussion history, and self/immediate family member migraine headache history. MAIN OUTCOME MEASURES: The odds of having a previous history of migraine headache were compared in the concussion group versus orthopedic controls. RESULTS: Controlling for between-group differences in age and sex, there was a significant positive association between concussion group status and history of migraine headache [adjusted odds ratio (OR), 1.90; 95\% confidence interval (CI), 1.03-3.50. P $=0.039$ ]. However, when including a previous concussion history in the statistical model, this relationship failed to reach significance [adjusted OR, 1.68; 95\% CI, 0.89-3.16. P = 0.107]. CONCLUSIONS: These results suggest that there is an association between migraine headache and concussion in athletes, but the cause-effect nature of this relationship cannot be determined. Migraine headache should be considered a modifying factor when caring for concussed athletes.

Comments. Migraine has been considered a modifying factor for concussion. Previous studies have shown a positive association between migraine and concussion, and that a previous diagnosis of migraine is associated with a prolonged recovery from traumatic head injury. ${ }^{1,2}$ This research study was case control observational study in young athletes. The population size was adequate with a sample size of 221 and the study design was quite simple. Essentially, migraine was found to be a risk factor for concussion, although the authors identify there is no way to determine causality from this data. The survey also detected an association between selfreport of previous concussion and migraine but lends itself to a discussion of whether migraine predisposes subjects to concussion symptoms following head trauma or previous concussion increases risk of migraine. Further data analysis found that approximately $80 \%$ of respondents with both previous migraine and concussion stated the concussion occurred prior to a clinical diagnosis of migraine. As with most trials of this nature, recall bias is certainly a limitation in this study. Historically, a clinical diagnosis of migraine is commonly erroneous, with patients typically under reporting migraine based on misdiagnosis as another headache subtype such as tension headache or sinus headache. Additionally, as the concussion group may have cognitive concerns that limit adequate history reporting. I suspect the finding in this study that migraine appears to be a modifying factor for concussion surprises none of our readership.

1. Kutcher JS, Eckner JT. At-risk populations in sports-related concussion. Curr Sports Med Rep. 2010;9(1):16-20.

2. Gordon KE, Dooley JM, Wood EP. Is migraine a risk factor for the development 
of concussion? Br J Sports Med. 2006;40(2):184-185.

-Wade M. Cooper, DO

Headache is the most common and frequently the most persistent symptom associated with mild traumatic brain injury (mTBI), or concussion. A number of risk factors have been reported for the development of post-concussive symptoms after mTBI, including history of prior concussion, personal or family history of migraine, and personal or family history of a mood disorder. ${ }^{1,2}$ Delayed symptom onset also appears to be an active predictor of continued post-concussive complaints. Since this particular study unfortunately did not control for one of these risk factors (prior concussion) while evaluating a second (history of migraine), conclusions from the results are limited.

1. Finnoff JT, Jelsing EJ, Smith J. Biomarkers, genetics, and risk factors for concussion. PM R. 2011;3(10 suppl 2):S452-S459.

2. Morgan CD, Zuckerman SL, Lee YM, King L, Beaird S, Sills AK, Solomon GS. Predictors of postconcussion syndrome after sports-related concussion in young athletes: a matched case-control study. J Neurosurg Pediatr. 2015;15:589-598.

-Robert G. Kaniecki, MD

The purpose of the study was to investigate the association between concussion and migraine. So this paper confirms the given knowns already referenced. That proof of causality was never to be an outcome was the study necessary? Fortunately, the subjects were put at no risk for harm so the study is not unethical, but I must be missing something the point because I struggle to understand anything new or importantly confirmatory. Seems to me confirmation of the association was already sufficient.

-Frederick R. Taylor, MD

\section{Friedman LE, Gelaye B, Bain PA, Williams MA. A Systematic Review and Meta-Analysis of Migraine and Suicidal Ideation. Clin J Pain. 2017;33:659-665.}

Abstract BACKGROUND: Previous studies have demonstrated an association between migraine and major depressive disorder. However, relatively little is known about the relationship between suicidal ideation, with or without concurrent depression, and migraine. OBJECTIVE: We conducted a systematic literature review to synthesize the available research focused on investigating the association of migraine with suicidal ideation. METHODS: Relevant research papers were identified through searches of major electronic databases including PubMed, Embase (Elsevier), Web of Science (Thomson Reuters), PsycINFO (EBSCO), and Google Scholar. We performed a meta-analysis to estimate the pooled unadjusted and adjusted odds ratios (ORs) and 95\% confidence intervals (95\% CI) for the association between migraine and suicidal ideation extracted from each study. RESULTS: A total of 148,977 participants in 6 studies were included in this analysis. Overall, findings from available studies documented elevated odds of suicidal ideation among individuals with migraines. In unadjusted 
models, the odds of suicidal ideation was 2.49-fold higher among individuals with migraine (OR, 2.49; 95\% CI, 2.34-2.65) compared with those without migraine. In multivariate-adjusted models, the pooled adjusted OR of suicidal ideation was 1.31 (OR, 1.31; 95\% CI, 1.10-1.55). CONCLUSIONS: A meta-analysis of available studies suggests a modest positive association between migraine and suicidal ideation. Further studies allowing for a more comprehensive investigation of the association between migraine and the full range of suicidal behaviors are warranted. A larger and more robust evidence-base may be useful to inform the clinical screening and diagnoses of comorbid conditions in migraineurs.

Comments. This abstract resonated with me. It is common for patients to describe feeling worn out by chronic migraine, and disclose in one way or another the idea of suicide to finally achieve mercy from their persistent symptoms. This abstract was a meta-analysis of suicide in patients with migraine and concluded those with migraine had a significantly higher rate of suicidal ideation. Common to meta-analysis type studies, the pooled number of subjects was impressive with 148,977 participants. The authors did not differentiate chronic migraine from episodic migraine in their analysis, which limits the ability to make inferences on intensity of migraine symptoms. My suspicion is that those with chronic migraine would be more likely to experience suicidal ideation. The authors discuss the possible association of genetic variants of serotonin transporter genes that are comorbid in migraine and depression as a potential mechanism for the increased risk. This seems a plausible component to their research findings. I was surprised to see the authors did not address the higher frequency of borderline personality disorders encountered within migraine populations. Borderline personality disorder has a prevalence of $2 \%$ prevalence in general, and is thought to be more common in those with migraine.

Additionally, patients with a diagnosis of borderline personality disorder were found to be more severely affected by migraine and more refractory to common migraine therapies. ${ }^{1,2}$ Patients with borderline personality disorder may experience increased suicidal ideation. The limits of this abstract include varying criteria for a diagnosis of migraine and suicidal ideation between the studies included in the meta-analysis. Also, the presence of active depression may have confounded the data such that the cross section analysis may not be truly representative of the risk for each patient over time.

1. Saper JR, Lake AE 3rd. Borderline personality disorder and the chronic headache patient: review and management recommendations. Headache. 2002;42(7):663-674.

2. Rothrock J, Lopez I, Zweilfer R, Andress-Rothrock D, Drinkard R, Walters N. Borderline personality disorder and migraine. Headache. 2007;47(1):22-26.

-Wade M. Cooper, DO

Evidence has established a higher prevalence of major depressive and bipolar disorders among those with migraine. Borderline personality disorder may also occur with greater frequency among those reporting migraine headaches. Since all of these mental health conditions have been clearly associated with increased rates of suicidal ideation, it should come as no surprise that migraine also carries such an association. The connections undoubtedly arise from neurobiological underpinnings but also are likely to involve the results of environmental stressors and life changes that impact these genetically predisposed brains. Those of us in Headache Medicine will be familiar with these genetic-environmental influences (epigenetic) in migraine activity, but I cite 2 articles adding perspectives from the psychiatry side. ${ }^{1,2}$

1. Rot M, Mathew S, Charney D. Neurobiological mechanisms in major depressive 
disorder. CMAJ. 2009;180:305-313

2. Mandelli L, Serretti A. Gene environment interaction studies in depression and suicidal behavior: an update. Neurosci Biobehav Rev. 2013;37:2375-2397.

-Robert G. Kaniecki, MD

While I could swear this migraine-suicidal ideation association has been known for decades, ${ }^{1,2}$ none of us in Headache Medicine (HM) will refer to Migraine as the Suicide Headache, Cluster already gets that nod. However, none of us in HM should ignore the known increased risk of suicide in migraine, and most certainly not when major depressive disorder or adverse life events coexist.

1. Breslau N, Davis GC, Andreski P. Migraine, psychiatric disorders, and suicide attempts: an epidemiologic study of young adults. Psychiatry Res. 1991;37:11-23.

2. Breslau N. Migraine, suicidal ideation, and suicide attempts. Neurology. 1992;42:392395.

-Frederick R. Taylor, MD

\section{Ruzieh M, Baugh A, Dasa O, Parker RL, Perrault JT, Renno A, Karabin BL, Grubb B. Effects of intermittent intravenous saline infusions in patients with medication-refractory postural tachycardia syndrome. $J$ Interv Card Electrophysiol. 2017;48:255-260.}

Abstract BACKGROUND: The postural tachycardia syndrome (POTS) is a heterogeneous group of disorders that results in symptoms of orthostatic intolerance. Excess blood pooling has been observed to cause low effective circulating volume in the central vasculature. Consequently, acute volume loading with IV saline has emerged as a potential strategy for clinical intervention. We evaluated the impact of acute volume loading on both the signs and symptoms of patients suffering from POTS. METHODS: Fifty-seven subjects screened from our population of POTS patients and assenting to participation were administered the two surveys by telephone. Subjects completed each survey twice, before, and after initiating IV hydration therapy. The Orthostatic Hypotension Questionnaire (OHQ) was used to assess change in clinical symptomatology, while the short form 36 health survey (SF-36) was employed to assess the impact of IV saline infusion on quality of life. RESULTS: Fifty-seven patients were included in the analysis. The average number of medications trialed before referral for IV hydration was $3.6 \pm 1.7$ medications. Saline infusions occurred with mean frequency of $11.3 \pm 8.5$ days and at a mean volume of $1.5 \pm 0.61$ per infusion. The mean change of the OHQ was $3.1 \pm 0.3$ (95\% CI 2.6-3.7; P $<0.001)$, with significant improvement in all the composite scores. The mean change in the SF-36 form was $19.1 \pm 2.7$ (95\% CI -24.6 to -13.6; P < 0.001). CONCLUSIONS: Intermittent IV infusions of saline dramatically reduce symptoms and improve quality of life in patients suffering from POTS. Further work should explore its efficacy as a bridge study for patients of high symptomatic severity.

Comments. Migraineurs have an increased prevalence of syncope and orthostatic intolerance compared to controls. ${ }^{1}$ The typical postural orthostatic tachycardia patient is slender, young females with enhanced autonomic activity which mimics the patient population at headache clinics. The experienced headache provider quickly becomes familiar with POTS. Patients may 
experience orthostatic headache associated with an exacerbation of their POTS related symptoms. $^{2}$ It is possible this association is due to fluid volume shifts and altered homeostasis that may trigger migraine. Some patients may experience hyperadrenergic symptoms with POTS, including sweating, tremor, and panic indicative of sympathetic overdrive which has also been implicated in migraine initiation. As such, patients with both POTS and migraine may be difficult to manage. This was an observational case control study for patients referred for hydration with a previous diagnosis of POTS. The inclusion criteria required at least one pharmacologic medication failure, with the average number of medications used prior to study entry being 3.6. The study consisted of mostly females (96\%) with an average age of 35 years. $24 \%$ of the study subjects had hypermobility. This creative study explored the value of normal saline infusion (between one and two liters) given approximately once every 1-2 weeks for acute exacerbation of POTS related symptoms. The orthostatic hypotension questionnaire (OHQ) was used to determine outcome, along with the quality of life metric SF-36. Patients appeared to respond amazingly well to this treatment, seen both in the $O H Q$ and SF-36. The typical duration of benefit was longer than 3 days, with continued protocol treatments weekly as needed. Of the 72 participants, 50 were able to discontinue treatment within 6 months. Only 4 patients reported no improvement in symptoms, 3 of which with hypermobility. Headache was not directly ascertained or monitored during the study which is truly unfortunate. I would have expected those with headache to have improved also. In general, saline infusion is quite safe and well tolerated. I have recently trialed this protocol in a few of my patients with both POTS and refractory chronic migraine with short term favorable response. I am curious to see how these patients do over time.

1. Thijs RD, Kruit MC, van Buchem MA, Ferrari MD, Launer LJ, van Dijk JG. Syncope in migraine: the population-based CAMERA study. Neurology. 2006;66(7):1034-1037.

2. Khurana RK, Eisenberg L. Orthostatic and non-orthostatic headache in postural tachycardia syndrome. Cephalalgia. 2011;31(4):409-415.

-Wade M. Cooper, DO

Acute volume loading in medically refractory POTS - one or two liters of saline every 1-2 weeks - apparently was beneficial in the short-term. Such a treatment seemingly would be safe and well-tolerated. Logistical and cost concerns are obvious. The study was open-label and uncontrolled, so results must be critiqued cautiously. Other means of volume expansion would seemingly be more cost-effective and more easily administered, so even if repetitive volume loading were of established benefit, it likely would be reserved for those failing simpler measures.

-Robert G. Kaniecki, MD

$W M C$ 's comment about the lack of ascertainment or monitoring for headache during the study is truly prescient. He might have written based on the Khurana and Eisenberg reference that the lack of ascertainment of migraine and association of occurrences of POTS and migraine was not only truly unfortunate, but a truly missed obligatory opportunity, or even a severe deficiency in study design. In the Khurana paper of 24 consecutive subjects with POTs, 23 (96\%) had migraine. While my experience is ripe with selection bias and most obviously anecdotal, POTS 
and migraine are inseparable and very disabling. So the study is interesting from the therapeutic viewpoint while I ditto many of $R G K^{\prime}$ 's concerns.

-Frederick R. Taylor, MD

\section{Parkitny L, Younger J. Reduced Pro-Inflammatory Cytokines after Eight Weeks of Low- Dose Naltrexone for Fibromyalgia. Biomedicines. 2017;5(2). pii: E16. doi: 10.3390/biomedicines5020016. PubMed PMID: 28536359.}

Abstract Fibromyalgia (FM) is a complex, multi-symptom condition that predominantly affects women. The majority of those affected are unlikely to gain significant symptomatic control from the few treatments that are approved for FM. In this 10-week, single-blind, crossover trial we tested the immune effects of eight weeks of oral administration of low-dose naltrexone (LDN). We enrolled eight women with an average age of 46 years, symptom severity of 62 out of 100 , and symptom duration of 14 years. We found that LDN was associated with reduced plasma concentrations of interleukin (IL)-1 $\beta$, IL-1Ra, IL-2, IL-4, IL-5, IL-6, IL-10, IL-12p40, IL-12p70, IL-15, IL-17A, IL-27, interferon (IFN)- $\alpha$, transforming growth factor (TGF)- $\alpha$, TGF- $\beta$, tumor necrosis factor (TNF)- $\alpha$, and granulocyte-colony stimulating factor (G-CSF). We also found a $15 \%$ reduction of FM-associated pain and an $18 \%$ reduction in overall symptoms. The findings of this pilot trial suggest that LDN treatment in fibromyalgia is associated with a reduction of several key pro-inflammatory cytokines and symptoms. The potential role of LDN as an atypical anti-inflammatory medication should be explored further.

Raknes G, Småbrekke L. Low-dose naltrexone and opioid consumption: a drug utilization cohort study based on data from the Norwegian prescription database. Pharmacoepidemiol Drug Saf. 2017;26:685-693.

Abstract PURPOSE: Low-dose naltrexone (LDN) is used in a wide range of conditions, including chronic pain and fibromyalgia. Because of the opioid antagonism of naltrexone, LDN users are probably often warned against concomitant use with opioids. In this study, based on data from the Norwegian prescription database, we examine changes in opioid consumption after starting LDN therapy. METHODS: We included all Norwegian patients $(\mathrm{N}=3775)$ with at least one recorded LDN prescription in 2013 and at least one dispensed opioid prescription during the 365 days preceding the first LDN prescription. We allocated the patients into three subgroups depending on the number of collected LDN prescriptions and recorded the number of defined daily doses (DDDs) on collected prescriptions on opioids, nonsteroidal anti-inflammatory drugs and other analgesics and antipyretics from the same patients. RESULTS: Among the patients collecting $\geq 4 \mathrm{LDN}$ prescriptions, annual average opioid consumption was reduced by 41 DDDs per person $(46 \%)$ compared with that of the previous year. The reduction was 12 DDDs per person $(15 \%)$ among users collecting two to three prescriptions and no change among those collecting only one LDN prescription. We observed no increase in the number of DDDs in nonsteroidal anti-inflammatory drugs or other analgesics and antipyretics corresponding to the decrease in opioid use. CONCLUSIONS: Possibly, LDN users avoided opioids because of warnings on concomitant use or the patients continuing on LDN were less opioid dependent than those terminating LDN. Therapeutic effects of LDN contributing to lower opioid consumption 
cannot be ruled out.

Comments. Naltrexone is a potent $\mu$-opioid and $\kappa$-opioid receptor antagonist that is FDA approved for treatment of opioid dependence and medication detoxification. Low dose naltrexone $(L D N)$ refers to the off label use of naltrexone in various clinical applications including multiple sclerosis, fibromyalgia and facial pain. The proposed mechanism for pain benefit is not completely understood. Currently concepts include the blocking of opioid receptors with low doses of naltrexone (typically between 1-4.5mg) resulting in increased production of endogenous endorphins for additional analgesia. Additionally, low dose naltrexone appears to have an antagonistic effect on toll like receptor IV (TLR-4) receptors found on the surface of macrophages, including glial cells within the central nervous system. ${ }^{1,2}$ Low dose naltrexone is generally well tolerated, with minimal side effects consisting of vivid dreams and fatigue. Generic naltrexone is usually available at low cost, however the smallest standard size is 50mg. Low dose naltrexone treatment is achieved either by splitting the generic tablet into small pieces which lends to inaccurate dosing, or obtaining the medication through a compounding pharmacy.

The first abstract comes from the same author who previously published on LDN in fibromyalgia. ${ }^{3}$ The reviewed abstract was a small trial in only 8 female subjects with a diagnosis of fibromyalgia by American College of Rheumatology Criteria (combination of widespread pain index and symptom severity scale - note tenderpoints no longer required). They had 2 weeks of no treatment then received $4.5 \mathrm{mg}$ of naltrexone (compounded) with the option to reduce to $3 \mathrm{mg}$ if they developed adverse events. Baseline inflammatory marker studies were obtained, followed by 8 weeks of therapy. At the end of 8 weeks, patients reported an overall improvement in fibromyalgia pain and overall symptoms. Serologies evaluated at the end of the 8 week trial showed significant reductions in several key inflammatory markers relevant to migraine and headache, including TNF- $\alpha$ and several interleukins. The obvious downside to this trial is small sample size and lack of a placebo. However, I was impressed with the overall reduction in inflammatory markers and feel this concept deserves a more robust exploration.

The second abstract is from a medication utilization database that examined prescription filling tendencies in Norway. Patients who were prescribed and received naltrexone were found to have a substantial reduction in opioid medication utilization, filling $46 \%$ less opioid medication than prior to being prescribed naltrexone. In comparison, patients prescribed NSAID class medication had no change in the quantity of opioid medication received over the same time. As an epidemiology study, one must be cautious of drawing too many inferences from the data. One pitfall of the study is not knowing if the reduction in opioid was indicative of improved pain state or instruction from the prescribing physician warning of co-administration of opioid with naltrexone. Regardless, this study essentially states patients who fill prescriptions for more than one year require less opioid medication over 12 months. What's not to like? I'm sure FRT or RGK will let me know.

1. Franchi $S$, Moretti $S$, Castelli $M$, et al. Mu opioid receptor activation modulates Toll like receptor 4 in murine macrophages. Brain Behav Immun. 2012 Mar;26(3):480-488

2. Selfridge BR, Wang X, Zhang Y, et al. Structure-Activity Relationships of (+)-NaltrexoneInspired Toll-like Receptor 4 (TLR4) Antagonists. J Med Chem. 2015 Jun 25;58(12):5038-5052.

3. Younger J, Noor N, McCue R, Mackey S. Low-dose naltrexone for the treatment of 
fibromyalgia: findings of a small, randomized, double-blind, placebo-controlled, counterbalanced, crossover trial assessing daily pain levels. Arthritis Rheum. 2013 $\mathrm{Feb} ; 65(2): 529-538$.

-Wade M. Cooper, DO

WMC listed the deficiencies of these 2 articles quite clearly. Article one has major flaws. Eight patients studied over 8 weeks? No blinding or placebo control? The second article has fewer limitations but one obvious failing. Common sense would dictate that most clinicians would decrease or eliminate opioid prescriptions when treating a patient with naltrexone. So WMC, that's what not to like.

-Robert G. Kaniecki, MD

Not so quick RGK. While neither study excites me, the later does confirm what is known from animal and human data; ${ }^{1,2}$ that is the combination of simultaneous use of low-dose naltrexone with most studied opioids improves analgesia, blunts physical dependence and at least a marker for psychological dependence. Furthermore, agonist-antagonist interactions can improve the therapeutic index of a single opioid at least as seen in animal models. ${ }^{2}$ Mechanistically it appears that combinations of opioid agonist or agonists--antagonist alter opioid functional selectively of $G$ proteins and/or $\beta$ arrestin-2 signaling and modulate regulators of $G$-protein signaling activity and/or downstream kinase activity caused by single ligands within the combination. ${ }^{2}$ In my database search I found a 1994 mouse study that helps me understand why simultaneous use may be successful. ${ }^{3}$ It's not uncommon practice in chronic pain units I am familiar with to use combinations including low-dose naltrexone based on the data already available. So while this second study confirms other data, nothing in the study is revelatory and most certainly not sensational or surprising, frankly any other result should have us questioning the study methodology. The lack of any NSAID benefit suggests lack of a necessary dose response effect given limited animal data. ${ }^{4}$

1. Chindalore VL, Craven RA, et al. Adding ultralow-dose naltrexone to oxycodone enhances and prolongs analgesia: a randomized, controlled trial of Oxytrex. J Pain. 2005; 6:392-399.

2. Davis MP. Evidence from basic research for opioid combinations. Expert Opin Drug Discov. 2012;7:165-178. doi: 10.1517/17460441.2012.648611.

3. Yoburn BC, Duttaroy A, Shah S, Davis T. Opioid antagonist-induced receptor upregulation: effects of concurrent agonist administration. Brain Res Bull. 1994;33:237240.

4. Miranda HF, Silva E, Pinardi G. Synergy between the antinociceptive effects of morphine and NSAIDs. Can J Physiol Pharmacol. 2004;82:331-338.

-Frederick R. Taylor, MD 


\section{Piper BJ, DeKeuster RM, Beals ML, Cobb CM, Burchman CA, Perkinson L, Lynn ST, Nichols SD, Abess AT. Substitution of medical cannabis for pharmaceutical agents for pain, anxiety, and sleep. J Psychopharmacol. 2017;31:569-575.}

Abstract A prior epidemiological study identified a reduction in opioid overdose deaths in US states that legalized medical cannabis (MC). One theory to explain this phenomenon is a potential substitution effect of MC for opioids. This study evaluated whether this substitution effect of MC for opioids also applies to other psychoactive medications. New England dispensary members $(n=1,513)$ completed an online survey about their medical history and MC experiences. Among respondents that regularly used opioids, over three-quarters $(76.7 \%)$ indicated that they reduced their use since they started MC. This was significantly $(p<0.0001)$ greater than the patients that reduced their use of antidepressants $(37.6 \%)$ or alcohol $(42.0 \%)$. Approximately two-thirds of patients decreased their use of anti-anxiety $(71.8 \%)$, migraine $(66.7 \%)$, and sleep $(65.2 \%)$ medications following MC which significantly $(p<0.0001)$ exceeded the reduction in antidepressants or alcohol use. The patient's spouse, family, and other friends were more likely to know about their MC use than was their primary care provider. In conclusion, a majority of patients reported using less opioids as well as fewer medications to treat anxiety, migraines, and sleep after initiating MC. A smaller portion used less antidepressants or alcohol. Additional research is needed to corroborate these self-reported, retrospective, cross-sectional findings using other data sources.

We are seeing a number of cannabis related publications making their way into peer reviewed literature. Some of these studies follow the same model as the selected abstract, in that they are surveys of people who are already using cannabis and frequenting a cannabis dispensary. In this case, the participants completed an online survey of medication utilization related to starting medical cannabis (MC). There are several major limitations to this type of data collection, with none larger than the selection bias of participants who are coming back to the store to purchase additional cannabis. I would assume those who do not respond would likely not come back to the dispensary to complete a survey. Additionally, the data was in large part retrospective with loads of recall bias. No surprise in that the patient's family was more likely to know of the MC use than the physician. In the state I work in where $M C$ is legal, some patients are quite hesitant to disclose their use of $M C$ in fear of ridicule or consequences of MC documentation in the medical chart. I agree that the finding of reduced opioid utilization in favor of MC is an interesting concept. If opioid use is consistently reduced in chronic pain patients using $M C$, one may see that as a step in the right direction. For now, the future of medical cannabis is hazy.

-Wade M. Cooper, DO

WMC began this month's discussion pitching fastballs, and clearly his arm grew tired. This last sequence of slow-pitch softballs has me yearning to discuss the hypothalamus again. Despite the biases clearly outlined by WMC, this article does provide some information to consider and discuss. It was interesting to see relatively greater reductions in opioid, migraine, and anxiety medications as compared to changes in antidepressant or alcohol use in patients on medical marijuana. Any reduction in opioid use and potentially opioid-related overdose deaths, if established, would indeed be quite meaningful. But such an optimistic view of the data and possible repercussions must be tempered by potential drawbacks not apparent in the dataset. 
Possible harms of habituation, tolerance, central nervous system functional and structural compromise must be considered for the individual, and in a migraine one must also contemplate an eventual medication-overuse effect - which I believe I have witnessed. Societal implications may be even more far-reaching. What we need is more data, more data, and more data.

-Robert G. Kaniecki, MD

On medical cannabis I am so very agreeable, not about its use (really uncomfortably wavering), but about the need for data. The experiment is now widely open in the public sphere and in every state where cannabis is legal for whatever use, scientists must feel obligated to collect data and publish it. All sorts of data, perhaps most especially long-term cognitive effects on the young brain. This may be the greatest human experiment of the 21 st century, if not any century, and we won't necessarily know whether that is simply hyperbole or apropos without the data, all the data, and more data.

-Frederick R. Taylor, MD

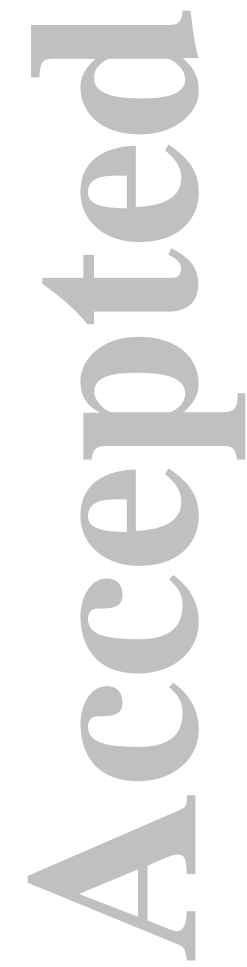

